\title{
Mucus plugs in patients with asthma linked to eosinophilia and airflow obstruction
}

\begin{abstract}
Eleanor M. Dunican, ${ }^{1}$ Brett M. Elicker, ${ }^{2}$ David S. Gierada, ${ }^{3}$ Scott K. Nagle, ${ }^{4}$ Mark L. Schiebler, ${ }^{4}$ John D. Newell, ${ }^{5}$ Wilfred W. Raymond, ${ }^{1}$ Marrah E. Lachowicz-Scroggins, ${ }^{1}$ Selena Di Maio, ${ }^{1}$ Eric A. Hoffman, ${ }^{5}$ Mario Castro, ${ }^{6}$ Sean B. Fain, ${ }^{4}$ Nizar N. Jarjour, ${ }^{7}$ Elliot Israel, ${ }^{8}$ Bruce D. Levy, ${ }^{8}$ Serpil C. Erzurum, ${ }^{9}$ Sally E. Wenzel, ${ }^{10}$ Deborah A. Meyers, ${ }^{11}$ Eugene R. Bleecker, ${ }^{11}$ Brenda R. Phillips, ${ }^{12}$ David T. Mauger, ${ }^{12}$ Erin D. Gordon, ${ }^{1}$ Prescott G. Woodruff, ${ }^{1}$ Michael C. Peters, ${ }^{1}$ John V. Fahy, ${ }^{1}$ and The National Heart Lung and Blood Institute (NHLBI) Severe Asthma Research Program (SARP) ${ }^{13}$

'Division of Pulmonary and Critical Care Medicine, Department of Medicine and Cardiovascular Research Institute, and 2Department of Radiology and Biomedical Imaging, UCSF, San Francisco, California, USA. ${ }^{3}$ Mallinckrodt Institute of Radiology, Washington University School of Medicine, St. Louis, Missouri, USA. ${ }^{4}$ Department of Medical Physics and Department of Radiology, University of Wisconsin School of Medicine and Public Health, Madison, Wisconsin, USA. 5Division of Cardiovascular and Pulmonary Imaging, Department of Radiology, University of lowa Carver College of Medicine, lowa City, lowa, USA. ${ }^{6}$ Division of Pulmonary and Critical Care Medicine, Department of Medicine, Washington University, St. Louis, Missouri, USA. ${ }^{7}$ Allergy, Pulmonary and Critical Care Medicine Division, University of Wisconsin School of Medicine, Madison, Wisconsin, USA. ${ }^{8}$ Pulmonary and Critical Care Medicine Division, Brigham and Women's Hospital, Boston, Massachusetts, USA. ${ }^{9}$ Department of Pathobiology, Lerner Research Institute, Cleveland Clinic, Cleveland, Ohio. ${ }^{10}$ Pulmonary, Allergy and Critical Care Medicine Division, Department of Medicine, University of Pittsburgh, Pittsburgh, Pennsylvania, USA. "Center for Genomics and Personalized Medicine Research, Wake Forest School of Medicine, Winston-Salem, North Carolina, USA. ${ }^{2}$ Division of Biostatistics and Bioinformatics, Department of Public Health Sciences, Penn State University, Hershey, Pennsylvania, USA. ${ }^{13}$ The NHLBI SARP investigators are detailed in the Supplemental Acknowledgments.
\end{abstract}

BACKGROUND. The link between mucus plugs and airflow obstruction has not been established in chronic severe asthma, and the role of eosinophils and their products in mucus plug formation is unknown.

METHODS. In clinical studies, we developed and applied a bronchopulmonary segment-based scoring system to quantify mucus plugs on multidetector computed tomography (MDCT) lung scans from 146 subjects with asthma and 22 controls, and analyzed relationships among mucus plug scores, forced expiratory volume in 1 second (FEV1), and airway eosinophils. Additionally, we used airway mucus gel models to explore whether oxidants generated by eosinophil peroxidase (EPO) oxidize cysteine thiol groups to promote mucus plug formation.

RESULTS. Mucus plugs occurred in at least 1 of 20 lung segments in $58 \%$ of subjects with asthma and in only $4.5 \%$ of controls, and the plugs in subjects with asthma persisted in the same segment for years. A high mucus score (plugs in $\geq 4$ segments) occurred in $67 \%$ of subjects with asthma with FEV 1 of less than $60 \%$ of predicted volume, $19 \%$ with FEV 1 of $60 \%-80 \%$, and $6 \%$ with FEV1 greater than $80 \%(P<0.001)$ and was associated with marked increases in sputum eosinophils and EPO. EPO catalyzed oxidation of thiocyanate and bromide by $\mathrm{H}_{2} \mathrm{O}_{2}$ to generate oxidants that crosslink cysteine thiol groups and stiffen thiolated hydrogels.

CONCLUSION. Mucus plugs are a plausible mechanism of chronic airflow obstruction in severe asthma, and EPO-generated oxidants may mediate mucus plug formation. We propose an approach for quantifying airway mucus plugging using MDCT lung scans and suggest that treating mucus plugs may improve airflow in chronic severe asthma.

TRIAL REGISTRATION. Clinicaltrials.gov NCT01718197, NCT01606826, NCT01750411, NCT01761058, NCT01761630, NCT01759186, NCT01716494, and NCT01760915.

FUNDING. NIH grants P01 HL107201, R01 HL080414, U10 HL109146, U10 HL109164, U10 HL109172, U10 HL109086, U10 HL109250, U10 HL109168, U10 HL109257, U10 HL109152, and P01 HL107202 and National Center for Advancing Translational Sciences grants UL1TR0000427, UL1TR000448, and KL2TR000428.

Related Commentary: p. 906

Conflict of interest: E.M. Dunican, B.M. Elicker, D.S. Gierada, S.K. Nagle, M.L. Schiebler, J.D. Newell, and J.V. Fahy are listed as inventors on a provisional patent application (W02017197360 A1) related to the development and application of the mucus score (Dunican score) as a biomarker and companion diagnostic tool in pulmonary disease. Role of funding source: This study was funded by the NIH and the National Center for Advancing Translational Sciences. The funding organization had no role in study design, data collection, analysis and interpretation of data, decision to publish, or preparation of the manuscript. 


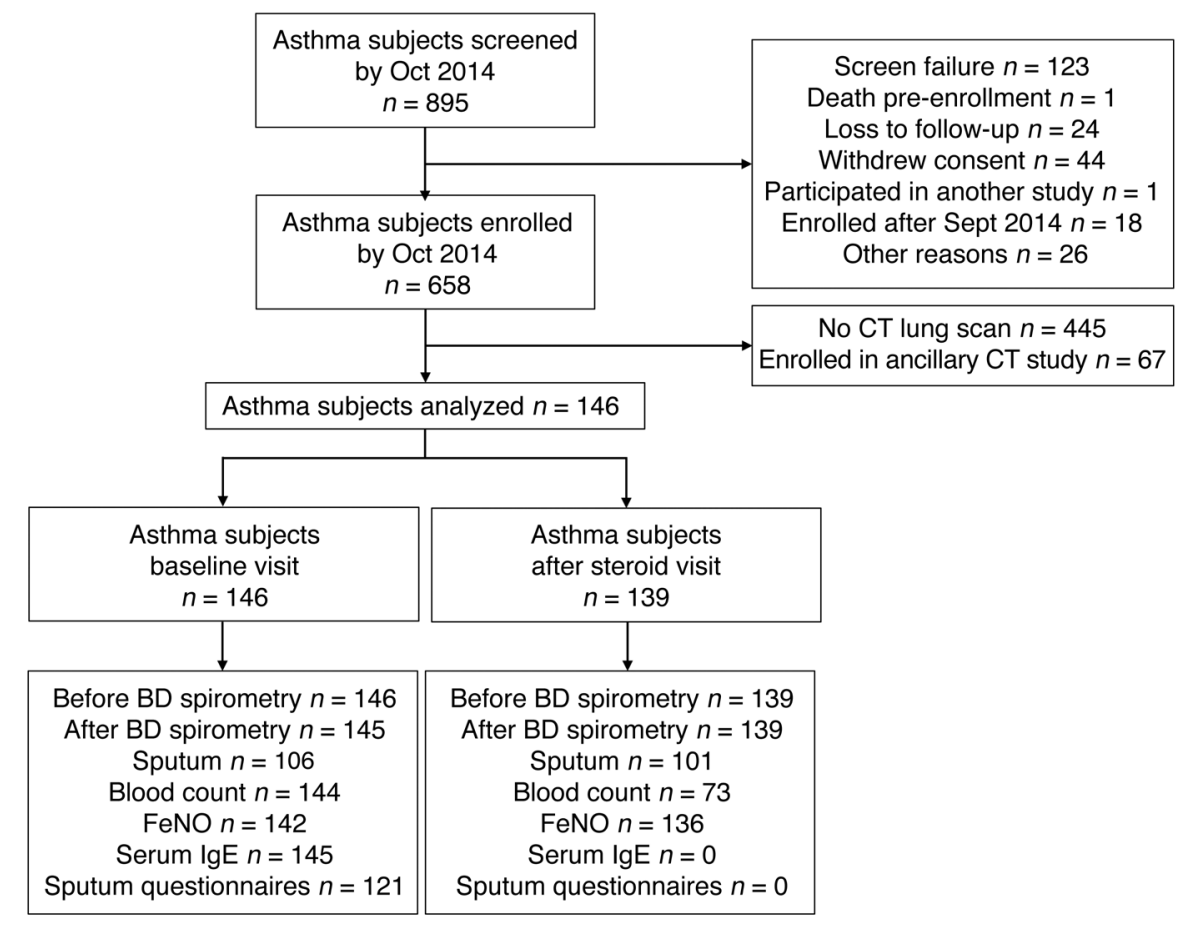

Figure 1. Consort diagram of CT substudy. Flow chart shows the number of asthma patients who were screened, enrolled, and included in the final analyses.

\section{Introduction}

Despite the prominence of mucus plugs in the pathophysiology of airflow obstruction in acute severe (fatal) asthma $(1,2)$, the role of mucus plugs in the pathophysiology of airflow obstruction in chronic severe asthma is poorly understood. This limited understanding is a barrier to rational treatment of airflow obstruction in severe asthma because mucus plugs represent a tractable treatment target if they can be shown to be a cause of obstruction. Developing an understanding of the role of mucus plugs as a mechanism of airflow obstruction in chronic severe asthma has been held back by methodologic difficulties. To date, imaging studies have not systematically examined the airways in patients with asthma for intraluminal mucus and studies that have documented a relationship between mucus pathology and airflow have relied on chronic cough and sputum production, a symptom complex known as chronic mucus hypersecretion $(\mathrm{CMH})(3,4)$. Reliance on $\mathrm{CMH}$ symptoms to identify patients with airway mucus plugs is problematic because $\mathrm{CMH}$ symptoms are often absent in patients with chronic obstructive pulmonary disease who have pathologically proven mucus plugs (5).

Pathology studies in small numbers of patients with nonfatal asthma reveal airway mucus plugs (6), but the relationship between these plugs and airflow obstruction is unclear. In addition, blood and airway eosinophilia are strongly correlated with airflow obstruction in asthma $(7,8)$, but the relationship between eosinophils and mucus plugs is unknown. Eosinophils secrete multiple biologically active molecules, including granule proteins, lipid mediators, chemotactic peptides, and cytokines. Eosinophil peroxidase (EPO) is the most abundant granule protein, and it utilizes respiratory burst-derived $\mathrm{H}_{2} \mathrm{O}_{2}$, halides (chloride and bro- mide), and pseudohalides (thiocyanate) to generate reactive oxidants that can kill pathogens or activate airway cells, including mast cells (9). Notably, the product of the reaction between thiocyanate and $\mathrm{H}_{2} \mathrm{O}_{2}$, catalyzed by EPO, is hypothiocyanous acid (HOSCN), which is known to target thiol groups as a cytotoxic mechanism (10). Recently, we have reported that oxidation of cysteine thiol groups - abundant in mucin polymers - is a mechanism of mucus gel stiffening in the lung (11), but the possibility that EPO promotes mucus plug formation by generating oxidants that modify mucins is not considered in reviews of the pathologic effects of eosinophils in the asthmatic airway (12).

We set out to examine the association between airway mucus plugs and airflow obstruction in chronic severe asthma and to explore whether eosinophils and EPO play a pathophysiologic role in the formation of these plugs. In clinical studies, we developed a scoring system to quantify mucus plugs in multidetector computed tomography (MDCT) scans of the lungs, and we used this MDCT-based scoring system to determine the relationship between mucus plugs and airflow obstruction in subjects with asthma in the SARP. We also examined the relationship between measures of mucus plugs and measures of airway eosinophils and EPO. In bench studies, we explored the pathologic effects of EPO in model systems of the airway mucus gel. Specifically, we tested to determine whether EPO catalyzes reactions of $\mathrm{H}_{2} \mathrm{O}_{2}$ with thiocyanate or bromide to generate oxidants that target cysteine thiols to crosslink them or that modify thiolated hydrogels to increase elasticity.

\section{Results}

Human subjects. MDCT scans of 146 adults with asthma and 22 healthy controls in the NHLBI SARP were analyzed (Figure 1 and Supplemental Table 1; supplemental material available online with this article; https://doi.org/10.1172/JCI95693DS1). Among the 146 patients with asthma, 66\% had disease qualifying as severe using American Thoracic Society/European Respiratory Society (ATS/ERS) criteria (Table 1) (13), and the prebronchodilator forced expiratory volume in 1 second (FEV1) was less than $80 \%$ of predicted volume in 97 patients $(66 \%)$ and less than $60 \%$ of predicted volume in 39 patients (27\%).

Airway mucus plugs can be identified and quantified using MDCT imaging of the lungs. In preliminary studies, we discovered that we could discern mucus plugs in the lungs of subjects with asthma using MDCT scans. Specifically, we could identify mucus plugs as areas of opacification within the airway lumen, contiguous with patent airway lumen across sequential transverse CT slices. These opacities were less radiodense than adjacent blood vessels, and occlusion of the lumen by these opacities could be partial or complete. These mucus plugs were predominantly seen 
Table 1. Characteristics of subjects with asthma across mucus score categories

\begin{tabular}{|c|c|c|c|c|}
\hline \multirow[t]{2}{*}{ Characteristic } & \multirow[t]{2}{*}{ All } & \multicolumn{3}{|c|}{ Mucus score } \\
\hline & & Zero & Low & High \\
\hline & $(n=146)$ & $(n=61)$ & $(n=45)$ & $(n=40)$ \\
\hline Mucus score & $0.5(0-4.5)$ & $0(0)$ & $1.5(0.5-2.5)$ & $9.5(6-12)$ \\
\hline Mean age $(y r)^{A}$ & $46.8 \pm 16.0$ & $43.2 \pm 15.4$ & $46.7 \pm 15.6$ & $52.3 \pm 16.3$ \\
\hline Female sex, no. (\%) & $91(62.3)$ & $43(70.5)$ & $26(57.8)$ & $22(55.0)$ \\
\hline Body mass index $\left(\mathrm{kg} / \mathrm{m}^{2}\right)$ & $32.7 \pm 9.3$ & $34.3 \pm 9.9$ & $32.5 \pm 10.5$ & $30.7 \pm 6.3$ \\
\hline \multicolumn{5}{|c|}{ Maintenance corticosteroid use, no. (\%) } \\
\hline Inhaled, any dose & $141(96.6)$ & $56(91.8)$ & $45(100.0)$ & $40(100.0)$ \\
\hline Inhaled, high dose $\mathrm{A}^{\mathrm{A}}$ & $102(69.9)$ & $36(59.0)$ & $30(66.7)$ & $36(90.0)$ \\
\hline Systemic ${ }^{A}$ & $15(10.3)$ & $3(4.9)$ & $3(6.7)$ & $9(22.5)$ \\
\hline $\mathrm{ACT}^{\mathrm{A}}$ & $18(14-21)$ & $19(15-21)$ & $18(14-22)$ & $16.5(13-19)$ \\
\hline Severe asthma, no. $(\%)^{A, B}$ & $96(65.8)$ & $31(50.8)$ & $29(64.4)$ & $36(90.0)$ \\
\hline \multicolumn{5}{|l|}{ Spirometry } \\
\hline FEV1 (\% of predicted volume) $)^{A, C}$ & $72.2 \pm 20.6$ & $81.0 \pm 16.2$ & $74.5 \pm 20.8$ & $56.1 \pm 17.4$ \\
\hline Sputum eosinophil count (\%) $)^{A, C, D}$ & $0.7(0,4.4)$ & $0.2(0,0.9)$ & $0.5(0.2,1.6)$ & $7.3(1.5,21.4)$ \\
\hline Blood eosinophil count $\left(\times 10^{6} /\right)^{\mathrm{AC}, \mathrm{CE}}$ & $306 \pm 276$ & $209 \pm 153$ & $309 \pm 282$ & $459 \pm 349$ \\
\hline FeNO (ppm) $)^{\mathrm{A}, \mathrm{F}}$ & $22(12,33)$ & $18(10,27)$ & $24(13,38)$ & $28(19,40)$ \\
\hline CMH, no. $(\%)^{\complement}$ & $41(34.0)$ & $18(29.5)$ & $10(22.2)$ & $13(32.5)$ \\
\hline Bronchiectasis on CT, no. (\%) & $29(19.9)$ & $7(11.5)$ & $11(24.4)$ & $11(27.5)$ \\
\hline \multicolumn{5}{|c|}{$\begin{array}{l}\text { Data reported as mean } \pm \mathrm{SD} \text { or median (IQR). Zero represents the mucus absent group (mucus score }=0 \text { ). Low } \\
\text { represents the group with mucus scores } 0.5-3.5 \text {, and high represents the group with mucus scores of } 4 \text { or more, } \\
\text { based on the median score of } 3.5 \text { in the mucus present group. }{ }^{A} P<0.05 \text { for comparison of zero and high scores. } \\
\text { BThe classification of asthma severity was determined using ATS/ERS criteria. }{ }^{C} P<0.05 \text { for comparison of low } \\
\text { and high scores. }{ }^{D} \text { Sputum cell counts were not available in } 40 \text { subjects due to ineligibility for sputum induction or } \\
\text { because the induced sputum did not meet quality metrics. }{ }^{E} \text { Blood measurements were not available for } 2 \text { subjects } \\
\text { FFeNO was not measured in } 4 \text { subjects. }{ }^{C} \text { Defined by WHO. Questionnaire data are missing in } 25 \text { patients (see } \\
\text { Supplemental Methods). }\end{array}$} \\
\hline
\end{tabular}

each subject. This approach generated scores ranging from 0 to 20 in increments of 0.5 . In this way, we found that mucus plugging was present in at least 1 of 20 lung segments in $58 \%$ $(85 / 146)$ of asthmatic subjects and in only $4.5 \%(1 / 22)$ of healthy controls (Figure 2E). The intraclass correlation coefficient (ICC) for between-rater mucus score agreement was 0.80 (95\% CI 0.74 to 0.85 ) for all 168 scans. In addition, the within-rater mucus score agreement for a random subset of 14 scans (3 healthy, 11 asthma) that was scored twice by each of the 5 radiologists was 0.99 (95\% CI 0.99 to 1.00). Among subjects with asthma, the median value of the mucus score in the mucus-present group was 3.5 , and we used this value to divide the asthmatic subjects into 3 mucus subgroups based on mucus score. Asthmatic subjects with a mucus score of 0 were assigned to the zero mucus group, while those with mucus scores between 0.5 and 3.5 were assigned to the low in subsegmental airways, appearing as focal or branching opacities (Figure 2, A-C), and usually occurred in the absence of bronchial dilatation. Based on these findings, we went on to develop a visual scoring system to formally quantify mucus plugs in MDCT scans (Figure 2D). Mucus plugs were defined as complete occlusion of a bronchus, irrespective of generation or size. When parallel to the scan plane, mucus plugs were recognized as tubular densities with or without branching. When oriented obliquely or perpendicularly to the scan plane, they were identified as oval or rounded opacities seen on sequential slices and differentiated from blood vessels by their continuity with patent portions of the bronchial lumen and their position relative to adjacent blood vessels (Supplemental Figure 1 and Supplemental Video 1). The segments of each lobe were systematically examined for the presence or absence of mucus plugs and given a score of 1 or 0 accordingly (Supplemental Figure 8). The segment scores of each lobe were summed to generate a total mucus score for both lungs, yielding an aggregate score ranging from 0 to 20. Peripheral airways within $2 \mathrm{~cm}$ of the diaphragmatic pleura and costal pleura were excluded from evaluation, as the small caliber of these peripheral airways makes occlusion by mucus difficult to ascertain. Five radiologists with subspecialty training in thoracic radiology reviewed the MDCT scans. Two radiologists were randomly assigned to score each scan independently, and the scores of both raters were averaged to generate the CT mucus score of mucus group and those with mucus scores between 4 and 20 were assigned to the high mucus group (Figure 2F).

Twenty-five asthmatic subjects in SARP 3 also had HRCT scans performed previously as part of SARP 1 or SARP 2 (Supplemental Table 2). These SARP 1 and SARP 2 scans were obtained 2 to 9 years prior to the SARP 3 MDCT scans. Two radiologists at the University of Wisconsin School of Medicine, Madison, center read the 50 scans together to identify and score the mucus plugs. In a score-based analysis, we compared mucus scores assigned to the first and second scans; mucus scores were unchanged in 7 subjects (28\%), increased in 10 subjects (40\%), and decreased in 8 subjects (32\%) over an average of 5.2 years (SD 2.5). We found that $90 \%$ of subjects with a high mucus score $(\geq 4)$ on the first scan had a high score on the second scan (Figure 2G). In a segment-based analysis, we compared individual lung segments in the first and second scans. Remarkably, $65 \%$ of lung segments that had a mucus plug on the first scan had a mucus plug in the same segment on the second scan. We also found that $80 \%$ of lung segments with no mucus plug on the first scan had no mucus plug in the same segment on the second scan (Figure 2, H and I). Persistent presence or absence of mucus plugs from first to second scan was seen with similar frequency across all bronchopulmonary segments (Supplemental Figure 2).

Airway mucus plugs usually occur in the absence of bronchiectasis. At the same time that the radiologists scored the scans for mucus 

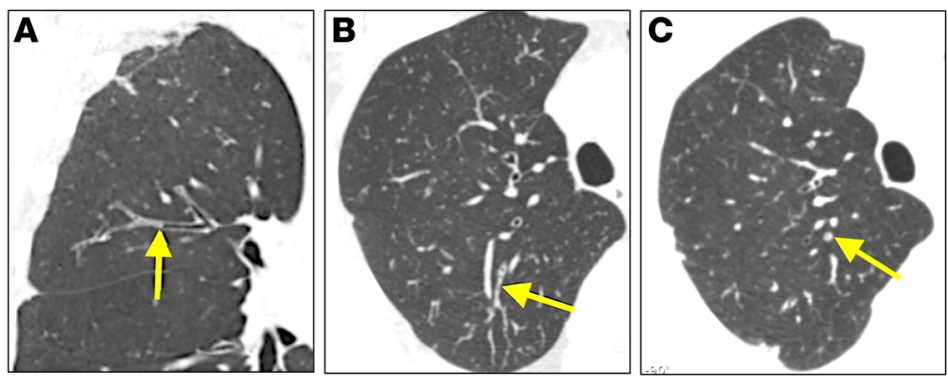
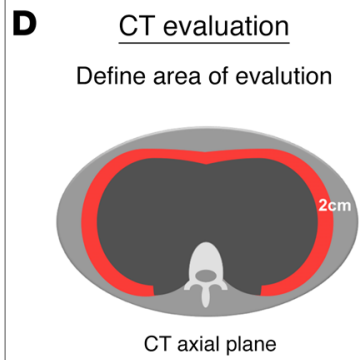

Exclude peripheral lung $\leq 2 \mathrm{~cm}$ from the costal or diaphragmatic pleura

from midline anteriorly to the mediastinal interface posteriorly

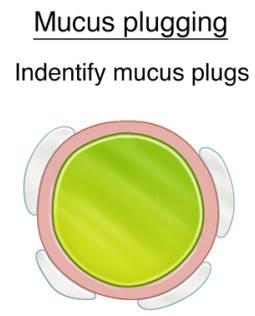

Plugged airway in cross-section

Mucus plug defined as complete occlusion of airway by mucus

Partial occlusion by mucus is not scored
Examine each bronchopulmonary segment for mucus plugs

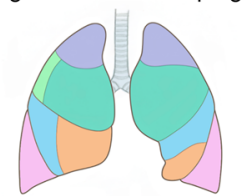

Bronchopulmonary segments

Count the bronchopulmonary segments with $\geq 1$ mucus plug(s) to generate the mucus score

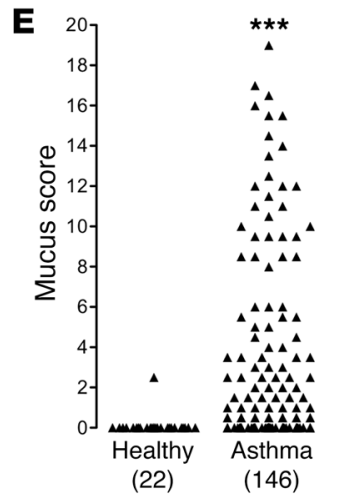

G

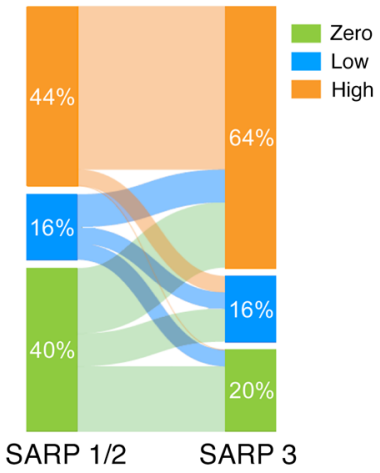

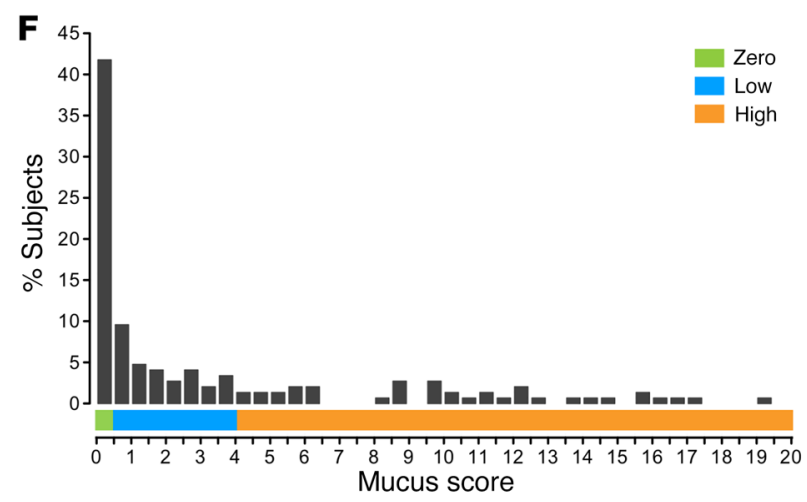

H

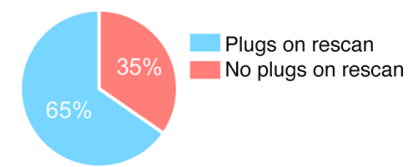

Segments with plugs

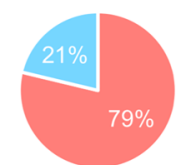

Segments with no plugs

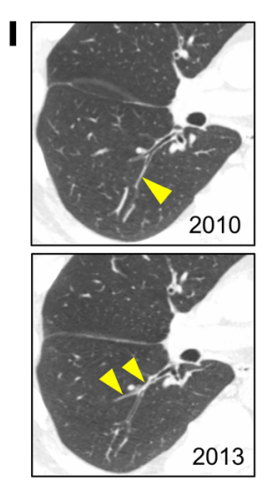

Figure 2. Development and distribution of the CT mucus score in asthma patients and healthy subjects. (A) Mucus plug with branching (yellow arrow) seen in longitudinal section is identified as a tubular opacification (frontal plane). (B) Mucus plug (yellow arrow) with extensive branching seen in longitudinal section (transverse plane). (C) Mucus plug (yellow arrow) seen in cross-section is identified as rounded opacification (transverse plane). (D) Schematic representation showing how MDCTs were evaluated to generate the mucus score. Airways within the $2 \mathrm{~cm}$ peripheral zone on MDCT (shown in red) or airways that were partially occluded were excluded from assessment. Mucus plugs were defined as complete occlusion of an airway. Each bronchopulmonary segment was assessed and scored for the presence or absence of 1 or more mucus plug(s), and the segment scores were summed to generate the mucus score. (E) Segment score in healthy patients and patients with asthma. (F) Frequency distribution of segment score in patients with asthma. The color code above the $x$ axis defines 3 mucus groups: green indicates patients with a mucus score of 0 (zero mucus group); blue indicates patients with mucus scores between 0.5 and 3.5 (low mucus group); and orange indicates patients with mucus scores of 4.0 or more (high mucus group). (C) Sankey bar graph showing the change in mucus score in 25 asthmatic subjects from SARP $1 / 2$ to SARP 3. (H) Pie chart of segments with mucus plugging on baseline scan; $65 \%$ of these segments had mucus plugging on rescan. Pie chart of segments with no mucus plugging on baseline scan; $79 \%$ of these segments had no mucus plugging on rescan. (I) MDCTs showing a mucus plug occluding the airway (yellow arrow) of the right lower lobe in 2010 and a mucus plug occluding the same airway, visible more proximally (yellow arrow) and branching into the adjacent airway, in 2013. ${ }^{* * *} P<0.001$, unequal variances $t$ test. plugging, they also systematically examined each of the 5 lung lobes for the presence or absence of bronchiectasis, defined as a bronchoarterial ratio of greater than 1.5. This approach generated a mean bronchiectasis score ranging from 0 to 5 as well as a binary outcome for presence or absence of bronchiectasis. We found that only $20 \%$ of the subjects with asthma had bronchiectasis (Table 1 and Figure 3A) and that bronchiectasis tended to be more frequent in subjects with a high mucus score than in those with a low or zero mucus score (Table 1$)(P=0.07)$. The prevalence of bronchiectasis or mucus plugging did not differ among the 5 lung lobes (Figure 3B), but the prevalence of mucus plugging was 4 to 5 times higher than that of bronchiectasis in any given lung lobe (Figure 3B). Bronchiectasis was weakly but significantly associated with mucus plugging at a lobar level - mainly because most lobes with bronchiectasis had mucus plugging (Figure 3C) - but relatively few lobes with mucus plugging also had bronchiectasis 

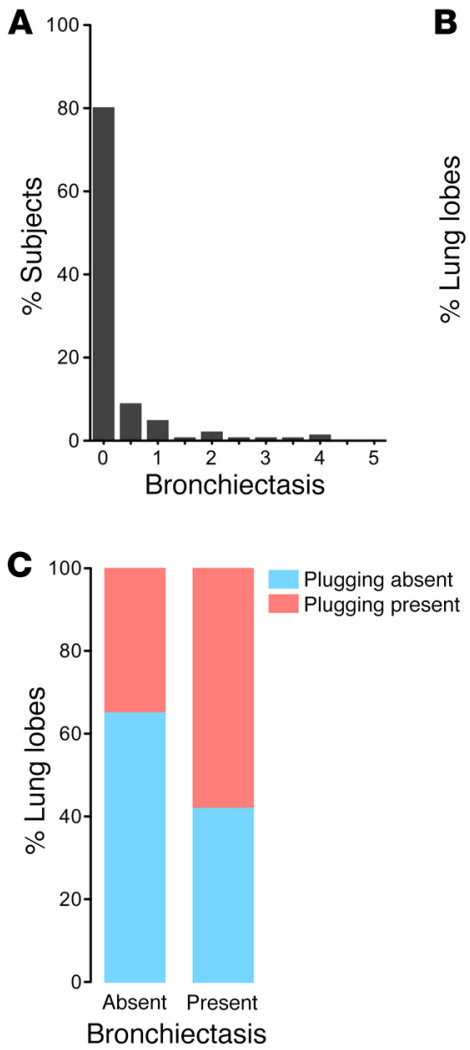
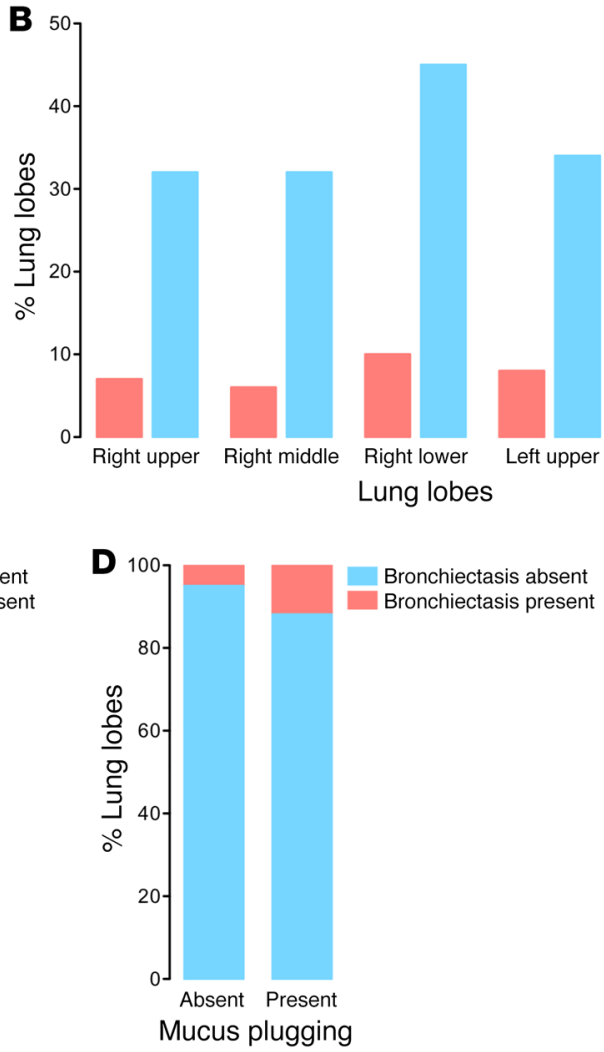

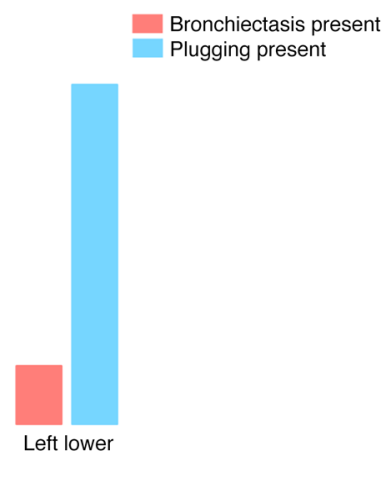

$\mathbf{E}$

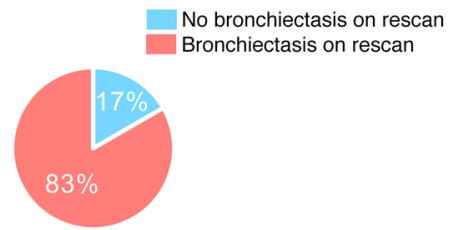

Lobes with bronchiectasis

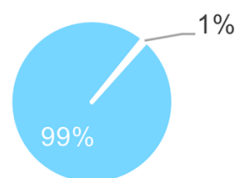

Lobes with no bronchiectasis

Figure 3. Relationship between bronchiectasis and mucus plugging. (A) Frequency distribution of bronchiectasis score in patients with asthma. (B) Prevalence of bronchiectasis versus mucus plugging in each lung lobe. The prevalence of mucus plugging is 4 to 5 times higher than the prevalence of bronchiectasis in each lobe. There is no significant difference in prevalence of bronchiectasis or mucus plugging across individual lobes. (C) Mucus plugging is present in 35\% of lobes that have no bronchiectasis present and $58 \%$ of lobes that have bronchiectasis present $(P=0.001)$. (D) Bronchiectasis is present in $5 \%$ of lobes that have no mucus plugging present and only $12 \%$ of segments that have mucus plugging present $(P=0.001)$. There is a positive association between mucus plugging and bronchiectasis, but mucus plugging usually occurs in the absence of bronchiectasis. (E) Pie charts illustrating the prevalence of bronchiectasis in repeat CT scans in 25 patients. The data show that $83 \%$ of lung lobes with mucus plugs visible on the first scan had mucus plugging visible on the second scan; in contrast, $99 \%$ of lung lobes with no mucus plugs visible in the first scan also had no mucus plugs visible on the second scan.

(Figure 3D). In an analysis of the 25 subjects with repeat MDCT scans for comparison, there was a low prevalence of mucus plugging on initial CT scan (2 out of 25 subjects) and the prevalence was unchanged in the second scan. In a lobe-based analysis, we compared individual lung lobes in the first and second scans and found that $83 \%$ of lung lobes (5/6 lobes) that showed bronchiectasis on the first scan showed bronchiectasis in the same lobe on the second scan. We also found that $99 \%$ of lung lobes (118/119 lobes) with no bronchiectasis on the first scan had no bronchiectasis in the same lobe on the second scan (Figure 3E).

Airway mucus plugs strongly associate with measures of airflow obstruction in asthma. To determine the association between airway mucus plugs and airflow obstruction in the subjects with asthma, we examined the relationship between the mucus score and measures of airflow obstruction by spirometry. We found that the mucus scores were inversely correlated with prebronchodilator measures of FEV1 percentage of predicted volume (Spearman's rho $=-0.51, P<0.001$ ), forced vital capacity (FVC) percentage of predicted volume (Spearman's rho $=-0.32, P<0.001$ ), and FEV1/FVC ratio of predicted volume (Spearman's rho $=-0.54, P<$ 0.001). These associations remained significant after controlling for age, sex, and measures of airway wall thickness in regression analyses (Table 2). The mean FEV1 was 25\% lower in the high mucus subgroup than in the zero mucus subgroup (Table 1 and Figure 4A), and the values for the FVC and FEV1/FVC ratio were also significantly decreased in the high mucus subgroup (Figure $4 \mathrm{~A})$. In addition, $66.7 \%$ of subjects with a prebronchodilator FEV1 of less than $60 \%$ of predicted volume had a high mucus score compared with $19 \%$ of subjects with FEV1 $60 \%-80 \%$ of predicted volume and $6.1 \%$ of subjects with FEV1 greater than $80 \%$ of predicted volume (Figure 4B). The low FVC in subjects with high mucus scores suggested air trapping in these subjects (14), and we confirmed this in a subset of subjects $(n=43)$ who had undergone body plethysmography as part of their baseline characterization studies. Specifically, we found that the ratio of residual volume to total lung capacity (RV/TLC) was also higher in the high mucus group than in the low mucus group, indicating more air trapping in the high mucus group $(P=0.04)$ (Supplemental Figure 3).

We next explored whether the strong association between MDCT mucus scores and abnormal lung function is reflected in abnormalities in other asthma outcomes. We found that, compared with the zero mucus group, the high mucus group had higher asthma medication requirements, worse asthma control test (ACT) scores, and were more frequently classified as having 
Table 2. Relationship among mucus score, spirometry measures, and sputum eosinophils (adjusted for covariates)

$\begin{array}{lccccc}\text { Asthma outcome }^{A} & \text { Unadjusted } & \text { Model 1 } & \text { Model 2 } & \text { Model 3 } & \text { Model } 4 \\ \text { FEV1, \% of predicted volume } & -2.2(-2.8,-1.5) & -1.9(-2.6,-1.3) & -1.9(-2.5,-1.2) & -1.5(-2.2,0.8) & -13(-21.5,-4.8) \\ & R^{2}=0.24, P<0.001 & R^{2}=0.28, P<0.001 & R^{2}=0.34, P<0.001 & R^{2}=0.38, P<0.001 & R^{2}=0.41, P=0.001 \\ \text { FVC, \% of predicted volume } & -1.3(-1.8 \text { to }-0.7) & -0.9(-1.5,-0.3) & -0.9(-1.4,-0.3) & -0.7(-1.3,-0.04) & -6.5(-14.4,1.4) \\ & R^{2}=0.11, P<0.001 & R^{2}=0.25, P=0.002 & R^{2}=0.27, P=0.003 & R^{2}=0.29, P=0.04 & R^{2}=0.30, P=0.1 \\ \text { FEV1/FVC } & -1.4(-1.8-1.0) & -1.5(-1.9,-1.1) & -1.4(-1.8,-1.0) & -1.3(-1.7,-0.8) & -8.0(-13,-2.7) \\ & R^{2}=0.26, P<0.001 & R^{2}=0.29, P<0.001 & R^{2}=0.34, P<0.001 & R^{2}=0.38, P<0.001 & R^{2}=0.41, P=0.003 \\ \text { Sputum eosinophils \% }{ }^{B} & 0.96(0.7,1.3) & 0.93(0.6,1.3) & 0.94(0.6,1.3) & \end{array}$

ALinear regression model reports $\beta$ coefficient $(95 \% \mathrm{Cl}$ ) for asthma outcomes. Predictor variable is mucus score ranging from 0 to 20 . Model 1 adjusts for the covariate of age at screening. Model 2 adjusts for the covariates of age and sex. Model 3 adjusts for the covariates of age, sex, and wall thickness percentage. Model 4 adjusts for the covariates of age, sex, and an interaction term for mucus score minus wall thickness percentage. ${ }^{B}$ Sputum data was missing in 40 patients.

severe asthma (Table 1 and Supplemental Methods). In addition, the percentage of patients in the high mucus group who had experienced at least 1 asthma exacerbation in the previous year was higher than in the zero mucus group, but this difference was not statistically significant (Supplemental Table 3). Notably, only 2 asthma subjects in the cohort met criteria for a diagnosis of allergic bronchopulmonary aspergillosis (ABPA) (15), and both had mucus scores in the low (0.5-3.5) range (Supplemental Table 3); sensitivity to other molds and aeroallergens did not differ significantly among mucus groups (Supplemental Table 4).

Influence of airway mucus plugs on treatment responses to $\beta$ adrenergic agonists and systemic corticosteroids. To explore whether airway mucus plugs influence treatment responses, we first examined responses to inhaled albuterol (540-720 mcg) using data from maximum bronchodilator reversibility testing (MBRT). Although there was no significant difference in the absolute change in FEV1 following albuterol treatment among the 3 mucus groups (Figure $5 \mathrm{~A}$ ), we found that the mean postbronchodilator FEV1 in the high mucus group was $23 \%$ lower than in the zero mucus group (Figure $5 \mathrm{~B}$ ) and that a persistently low FEV1 (FEV1 $<80 \%$ of predicted volume) following MBRT was common in subjects with a high mucus score, but uncommon in subjects with a zero mucus score (Figure 5C).

We next examined responses to intramuscular triamcinolone acetonide $(40 \mathrm{mg})$ using data from systemic corticosteroid responsiveness testing (SCRT). Although there was no significant difference in the absolute change in FEV1 following corticosteroid treatment among the 3 mucus groups (Figure 5D), we found that the mean poststeroid FEV1 was 20\% lower in the high mucus group than in the zero mucus group (Figure 5E). As with the data for albuterol treatment, we noted that a persistently low FEV1 following SCRT was common in subjects with a high mucus score, but uncommon in subjects with a zero mucus score (Figure

Figure 4. Mucus plugging is associated with low lung function. (A) Spirometric measures of lung function (FEV1, FVC, and FEV1/FVC) in the subjects with a high mucus score were significantly lower than in subjects with a low mucus score and subjects with a zero mucus score. (B) High mucus plug scores were much more common in patients with severe airflow obstruction. ${ }^{* *} P<0.001 ;{ }^{* *} P<0.01$, Kruskal-Wallis test with Dunn's correction.
$5 \mathrm{~F})$. In addition, we found that the CT mucus score was an independent predictor of residual abnormalities in FEV1 after systemic corticosteroid administration in logistic regression models (Supplemental Figure 4).

Finally, we examined responses to combined treatment with albuterol and systemic corticosteroid among the 3 mucus subgroups. Here, we found that the absolute change in FEV1 following MBRT and SCRT was significantly higher in the high mucus group than in the zero and low mucus groups (Figure 5G), but the mean postbronchodilator/poststeroid FEV1 was still significantly lower in the high mucus group than in the zero mucus group (Figure $5 \mathrm{H}$ ). Half of the subjects with high mucus scores had persistently low

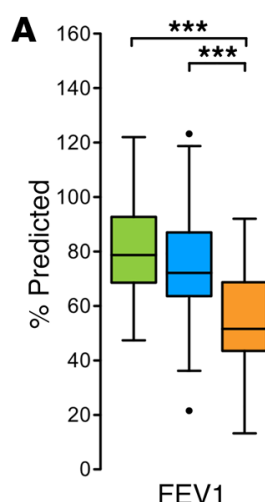

FEV1

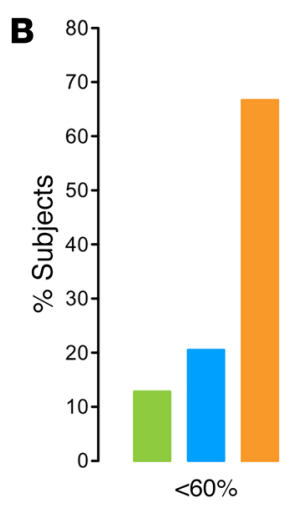

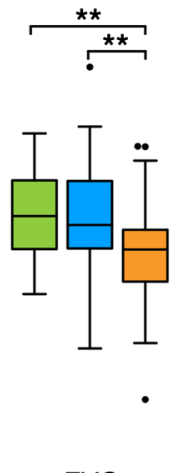

FVC
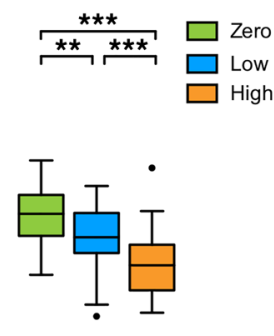

FEV1/FVC 


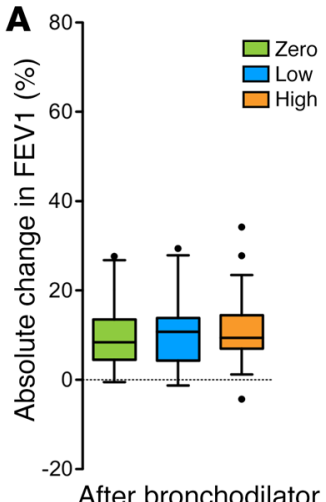

After bronchodilator
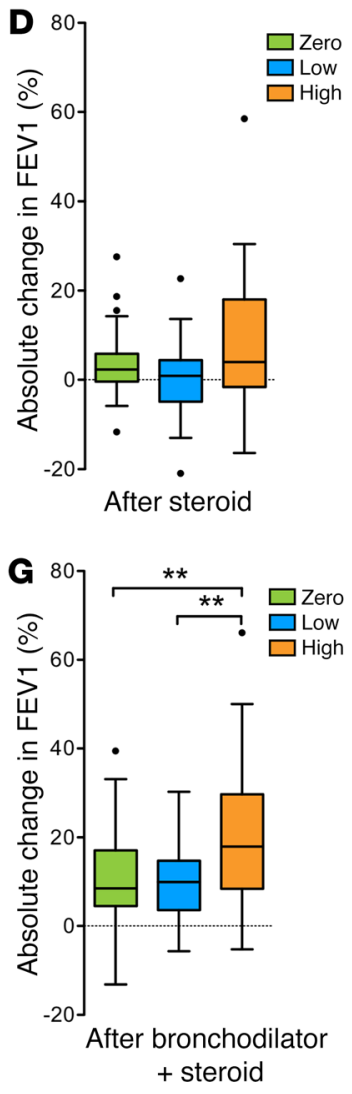
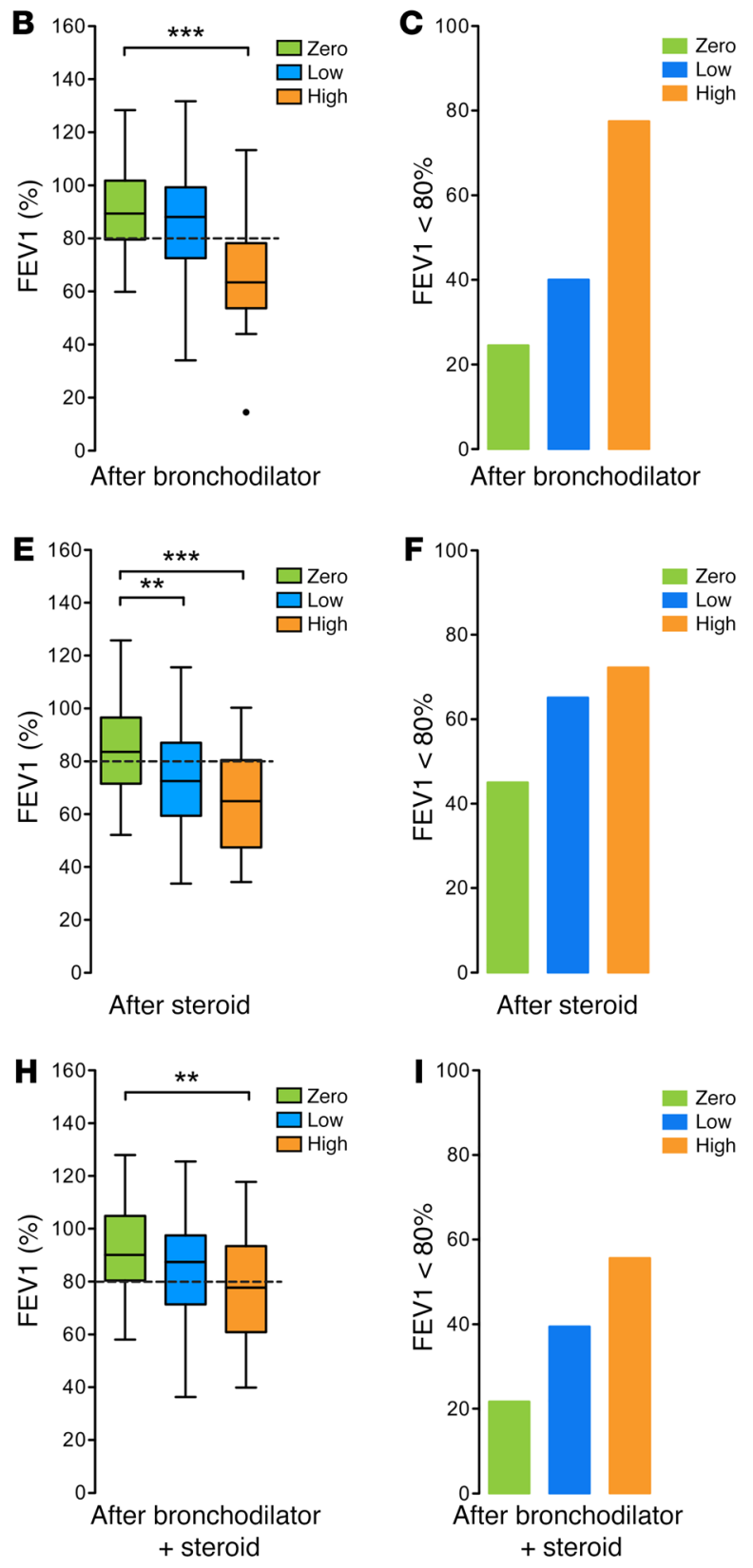

Figure 5. Persistent airflow obstruction is seen in subjects with high mucus scores after treatment with bronchodilators and steroids.

(A) The absolute change in FEV1 percentage of predicted volume after bronchodilator treatment did not differ across mucus groups. (B) The FEV1 percentage of predicted volume after bronchodilator treatment was significantly lower in the high mucus group than in the zero mucus group. (C) Residual postbronchodilator abnormalities in FEV1 (FEV1 < 80\%) occur more commonly in subjects with a high mucus score than in those with a zero mucus score.

(D) The absolute change in FEV1 percentage of predicted volume after steroid treatment did not differ across mucus groups. (E) The FEV1 percentage of predicted volume after steroid treatment was significantly lower in the high and low mucus groups than in the zero mucus group. (F) Residual poststeroid abnormalities in FEV1 (FEV1 < 80\%) occurred more commonly in subjects with a high mucus score than in those with a zero mucus score. (C) The absolute change in FEV1 percentage of predicted volume after bronchodilator and steroid treatment was significantly higher in the high mucus group than in the zero mucus group. (H) The FEV1 percentage of predicted volume after bronchodilator and steroid treatment was significantly lower in the high mucus group than in the zero mucus group. (I) Residual postbronchodilator and poststeroid abnormalities in FEV1 (FEV1 $<80 \%$ ) occurred more commonly in subjects with a high mucus score than in those with a zero mucus score. ${ }^{* *} P<0.001 ;{ }^{* *} P<0.01$ Kruskal-Wallis test with Dunn's correction.
FEV1 following SCRT and MBRT, whereas only a small subgroup of subjects with zero mucus scores had persistently low FEV1 (Figure 5I). Thus, aggressive treatment with $\beta$ adrenergic agonists and corticosteroids frequently does not normalize lung function in subjects with airway mucus plugs, and additional treatments need to be considered for these subjects.

Symptoms of $\mathrm{CMH}$ are neither sensitive nor specific for mucus plugs. To determine whether subjects with asthma could have mucus plugs without $\mathrm{CMH}$ symptoms, we examined the frequency of symptoms of $\mathrm{CMH}$ in the 3 mucus plug subgroups. Among 121 subjects who completed the cough and sputum questionnaire, 41 (34\%) satisfied WHO criteria for $\mathrm{CMH}$ (cough and sputum production on most days for at least 3 months a year for at least 2 consecutive years) (16). We found that 16 (40\%) subjects in the high mucus group did not have symptoms of CMH (Table 1). Converse- ly, we found that 18 (30\%) subjects in the zero mucus group had symptoms of $\mathrm{CMH}$. Although the subgroup of subjects with $\mathrm{CMH}$ did not have higher mucus scores than subjects without $\mathrm{CMH}$, the subjects with $\mathrm{CMH}$ were characterized by other clinical differences, such as older age, higher BMI, and evidence of more severe asthma (Supplemental Table 5). Interestingly, subjects with $\mathrm{CMH}$ did not differ from subjects without $\mathrm{CMH}$ in blood or sputum cell differentials or in sputum cell gene expression of cytokines or mucin genes (Supplemental Table 5).

Mucus plugging shown on MDCT scans is associated with airway eosinophilia. To explore whether eosinophils play a pathophysiologic role in the formation of mucus plugs, we analyzed multiple outcomes related to type 2 inflammation in blood and biospecimens in the 3 mucus subgroups. We found that eosinophils in blood and sputum and nitric oxide levels in exhaled breath were 

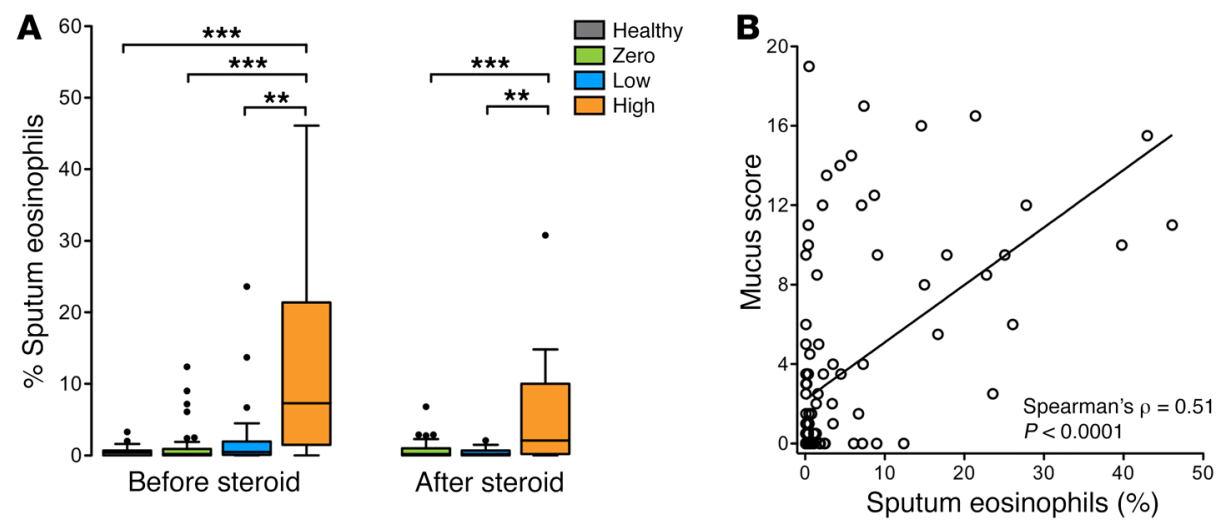

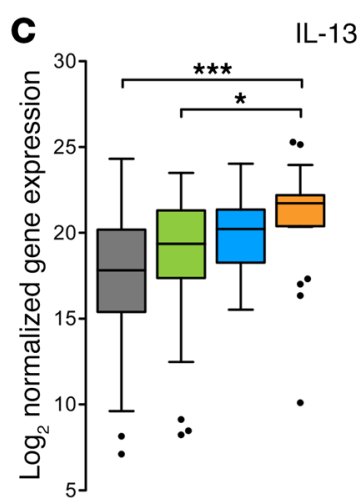

Before steroid
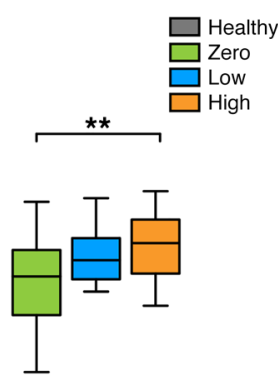

After steroid
$\mathbf{E}$

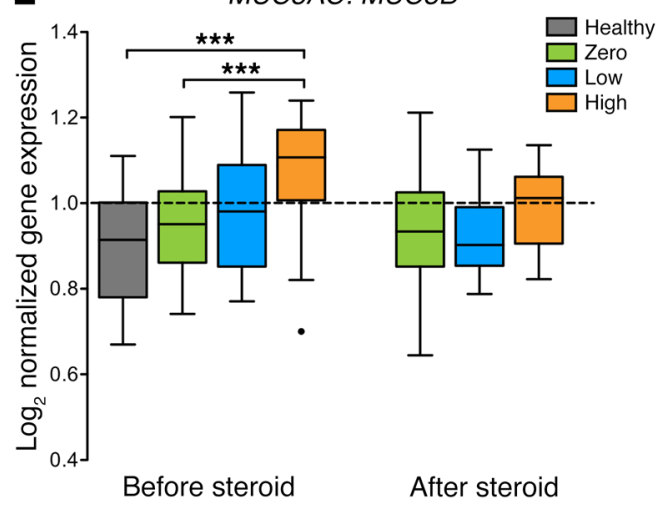

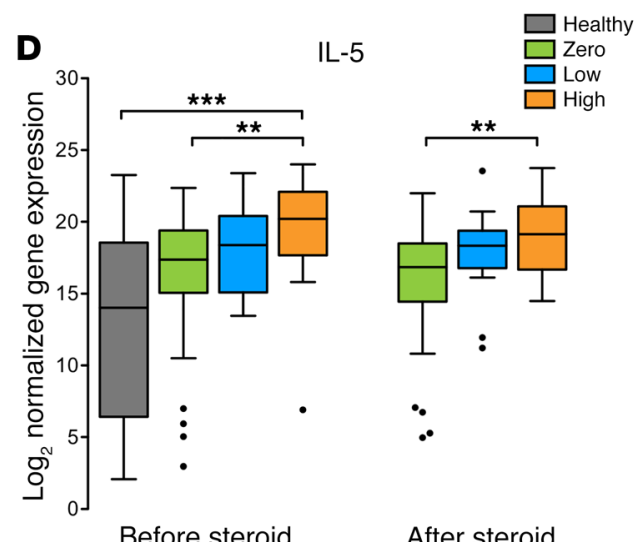

Before steroid After steroid

Figure 6. High mucus score is associated with markers of type $\mathbf{2}$ inflammation. (A) Sputum eosinophil percentage is significantly increased in patients with a high mucus score and remains significantly increased in patients with a high mucus score following treatment with intramuscular steroid (triamcinolone acetonide). (B) The sputum eosinophil percentage is significantly and positively associated with the mucus score. (C) Gene expression for IL-13 is significantly increased in patients with a high mucus score and remains significantly increased in patients with a high mucus score following treatment with intramuscular steroid. (D) Gene expression for IL-5 is significantly increased in patients with a high mucus score and remains significantly increased in patients with a high mucus score following treatment with intramuscular steroid. (E) The MUC5AC/MUC5B ratio is significantly increased in patients with high mucus scores. ${ }^{* *} P<$ $0.001 ;{ }^{*} P<0.01 ;{ }^{*} P<0.05$. $P$ values were determined by Kruskal-Wallis test with Dunn's correction unless otherwise indicated.

groups. We found that gene expression for IL-13 and IL-5 in sputum cells was significantly higher in the high mucus group than in the low and zero mucus groups and remained high following systemic corticosteroid treatment (Figure 6, C and D). In addition, we found that the ratio of gene expression of MUC5AC to MUC5B in sputum cells was significantly higher in the high mucus group than in the low and zero mucus groups and normalized following systemic corticosteroid treatment (Figure 6E). The presteroid pattern of a high expression of MUC5AC relative to MUC5B in the high mucus group is typical of the activation effects of IL-13 on mucin expression by airway epithelial cells (17).

EPO generates oxidants that crosslink cysteines and stiffen thiolated hydrogels. We have recently shown that neutrophil-driven oxidation crosslinks cysteine-rich mucin polymers to stiffen airway mucus gels in cystic fibrosis (11), and we explored here whether eosinophil-driven oxidation might be a mechanism of mucus 

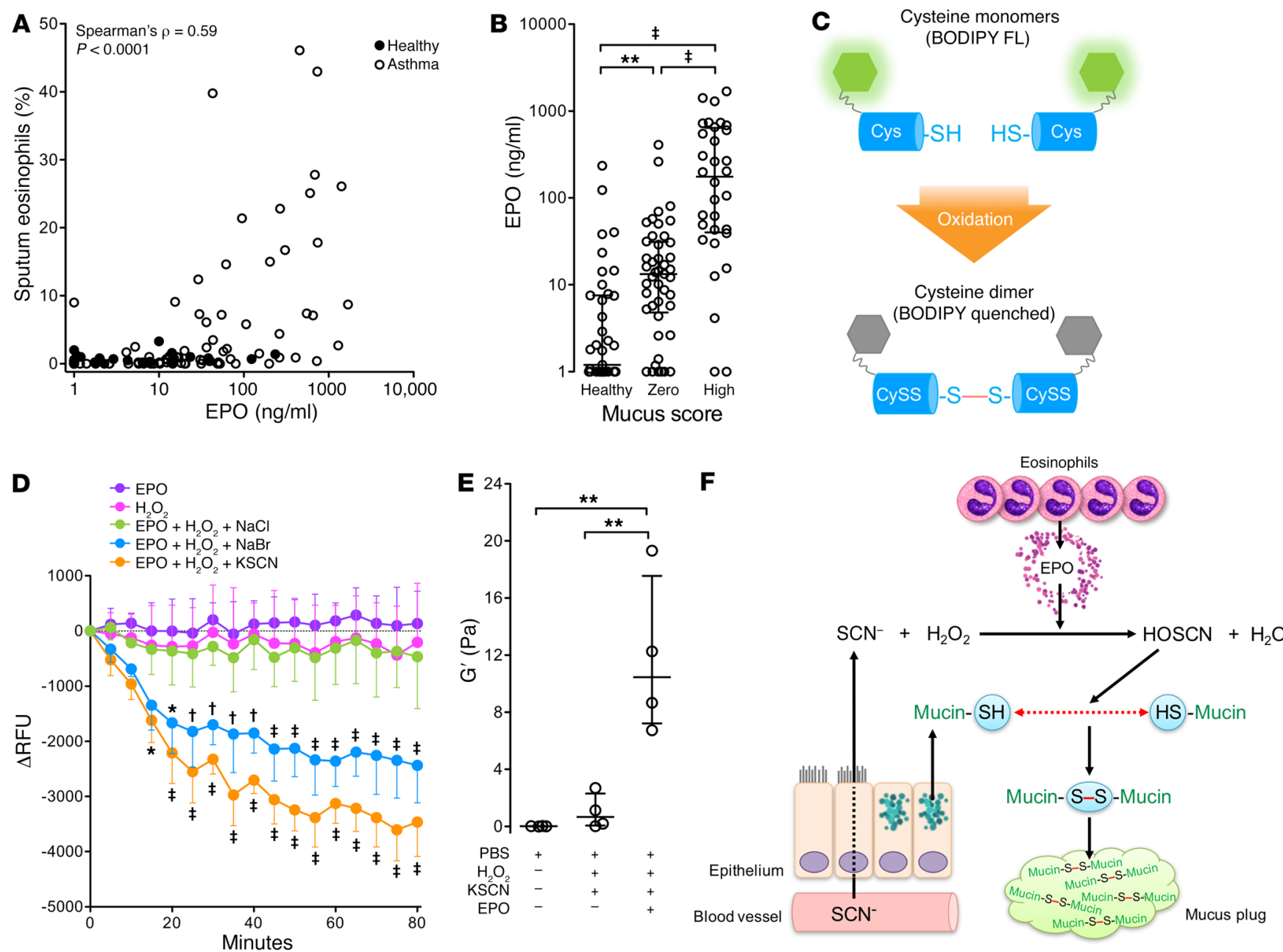

Figure 7. Eosinophil products are associated with mucus plugging. (A) The sputum eosinophil percentage is positively associated with sputum EPO levels. (B) Sputum EPO is higher in the high mucus group $(n=32)$ than in the zero mucus group $(n=45)$ and healthy controls $(n=39)$. (C) Schematic representation of the cysteine-linking assay: 2 cysteines labeled with BODIPY FL fluoresce green as monomers but quench when oxidized to form a cystine dimer. (D) Effect of EPO and $\mathrm{H}_{2} \mathrm{O}_{2}$ on cysteine crosslinking in the presence of chloride, bromide, or thiocyanate. Cysteines do not undergo significant crosslinking with EPO and $\mathrm{H}_{2} \mathrm{O}_{2}$ in the presence of chloride, but cysteines exposed to EPO and $\mathrm{H}_{2} \mathrm{O}_{2}$ in the presence of bromide, and especially thiocyanate, undergo much more oxidation and crosslinking. RFU, relative fluorescent units. (E) Effect of HOSCN, the product of EPO-catalyzed reaction of $\mathrm{H}_{2} \mathrm{O}_{2}$ and thiocyanate, on the viscoelastic properties of a thiolated hydrogel measured by rheology. A large increase in the elastic modulus ( $\mathrm{C}^{\prime}$ ) of the hydrogel was seen following exposure of the hydrogel to EPO with $\mathrm{H}_{2} \mathrm{O}_{2}$ and KSCN. There was no significant increase in $\mathrm{G}^{\prime}$ in the hydrogel in the absence of EPO. (F) Conceptual model for how type 2 inflammation promotes airway mucus plug formation in asthma. IL-13 increases thiocyanate transfer into the airway lumen. Once in the airway lumen, it is oxidized by $\mathrm{H}_{2} \mathrm{O}_{2}$ to form $\mathrm{HOSCN}$, a reaction catalyzed by EPO. HOSCN targets cysteine thiol groups in secreted mucin polymers to generate covalent disulfide mucin crosslinks. Crosslinked mucins have a high elasticity that decreases their clearance by the mucociliary escalator and results in mucus plug formation. The data are presented as mean \pm SD of 3 replicates in $\mathbf{D}$ and 4 replicates in $\mathbf{E} .{ }^{*} P<0.05 ;{ }^{*} P<0.01 ;{ }^{\dagger} P<0.01 ;{ }^{\ddagger} P<0.001$. $P$ value was determined by ANOVA with Bonferroni's correction.

plug formation in asthma. The activity of EPO can generate products that target thiol groups as a cytotoxic mechanism (10), and we considered the possibility that EPO-generated products oxidize the thiol groups of cysteine residues in mucin polymers to cause mucus plugs to form. As a first step, we measured EPO in sputum from the zero and high mucus subgroups and healthy controls. We found a positive correlation between sputum EPO levels and sputum eosinophils (Spearman's rho $=0.59, P<0.001$ ) (Figure 7A) and also found that EPO levels in the high mucus plug group were markedly higher than in the zero mucus group and in healthy controls (Figure 7B). We next tested the effect of EPO activity on cysteine crosslinking using 2 model systems of the airway mucus gel. The first model was a boron-dipyrromethenelabeled (BODIPY-labeled) cysteine reagent that quenches when cysteine forms its oxidized disulfide product (cystine) (Figure 7C). The second model system was a synthetic thiolated hyaluronan reagent that forms a hydrogel and increases its elasticity when oxidized (18). EPO catalyzes the oxidation of chloride, bromide, and thiocyanate by $\mathrm{H}_{2} \mathrm{O}_{2}$ to hypochlorous acid ( $\mathrm{HOCl}$ ), hypobromous acid ( $\mathrm{HOBr}$ ), and HOSCN, respectively. Specificity constants indicate that thiocyanate is a major substrate for EPO, and HOSCN is known to be a more thiol-specific oxidant than $\mathrm{HOBr}$ or HOCl (10). To test whether EPO generates HOSCN that catalyzes conversion of cysteine to its oxidized disulfide product 
(cystine), we exposed BODIPY-labeled cysteine to EPO in the presence of $\mathrm{H}_{2} \mathrm{O}_{2}$ and either chloride $(\mathrm{NaCl})$, bromide $(\mathrm{NaBr})$, or thiocyanate (KSCN). We found no significant cystine dimer formation when BODIPY-labeled cysteine was exposed to EPO, $\mathrm{H}_{2} \mathrm{O}_{2}$, and chloride, but dimer formation was significantly greater when the BODIPY-labeled cysteine was exposed to EPO, $\mathrm{H}_{2} \mathrm{O}_{2}$, and bromide and was greatest when the BODIPY-labeled cysteine was exposed to EPO, $\mathrm{H}_{2} \mathrm{O}_{2}$, and thiocyanate (Figure 7D). Thus, $\mathrm{HOBr}$ and HOSCN can oxidize cysteines, but HOSCN is more potent and $\mathrm{HOCl}$ has no effect in this system. We next tested to determine whether HOSCN can crosslink the thiolated hyaluronan reagent. Here, we used a cone and plate rheometer to measure changes in the elasticity of the hydrogel under different conditions. We found that the combination of EPO, $\mathrm{H}_{2} \mathrm{O}_{2}$, and thiocyanate markedly increased the elasticity of the thiolated hyaluronan gel and that EPO was required for this effect (Figure 7E). Thus, HOSCN can convert a thiolated hydrogel from a liquid form to a solid form, leading us to propose that HOSCN is an oxidant product of EPO activity that may mediate mucus plug formation in asthma (Figure 7F).

Nearly $60 \%$ of the subjects in the high mucus subgroup had reported an asthma exacerbation in the previous year, but nearly $50 \%$ of the zero mucus group had also experienced an asthma exacerbation in that time frame. The $20 \%$ increase in exacerbations in the high versus zero mucus groups did not reach statistical significance in our cohort, and larger studies than ours will be needed to more fully explore the relationship between airway mucus plugs and exacerbations in severe asthma.

\section{Discussion}

Among the asthmatic subjects we studied, $66 \%$ had physiologic evidence of airflow obstruction and $27 \%$ had severe obstruction. Using a method of quantifying mucus plugs based on visual assessment of MDCT scans by experienced chest radiologists, we found a strong inverse relationship between the MDCT mucus score and FEV1 values. We also found that the majority of subjects with severe obstruction had at least 4 bronchial subsegments with 1 or more airways completely occluded with mucus. Some of these subjects had mucus plugs in more than half of their bronchial subsegments. Although strong associations do not prove causality, we propose that the association between mucus plugs and airflow obstruction in these subjects with asthma is causal because mucus plugs that completely occlude subsegmental airways will cause regional airflow obstruction at a minimum and more widespread airflow limitation when present in multiple bronchopulmonary segments. Indeed, we found that subjects with high mucus scores had air trapping, as evidenced by low FVC and high RV. Air trapping is recognized as a prominent characteristic of severe forms of asthma, but the mechanisms have not been well understood (14). The mucus plugs we identify here represent a plausible mechanism of air trapping in at least some of the subjects who have this physiologic abnormality.

Although the mucus plugs were heterogeneously distributed in the 20 bronchopulmonary segments among subjects, we found that they tended to be found in the same bronchopulmonary segment within individual subjects over long periods of time. The mechanism of this susceptibility to mucus plug formation in specific airways is not anatomical because bronchiectasis was an uncommon finding in our patients and mucus plugs usually occurred in the absence of bronchiectasis. We consider it unlikely that the same mucus plug is long lived for years in an airway and more likely that mucus plugs repeatedly form and reform in susceptible bronchopulmonary segments. We speculate that these segments are susceptible to mucus plugging because they have heightened type 2 inflammation based on associations we found between high mucus plug scores and increased sputum measures of eosinophils and type 2 cytokines.

The protocol followed by participants in SARP included a maximal bronchodilator reversibility test and a systemic corticosteroid response test. Data from these tests allowed us to comprehensively assess the effect of mucus plugs on treatment responses to inhaled albuterol and systemic triamcinolone. We discovered that subjects in the high mucus subgroup who had marked reduction in FEV1 and FVC pretreatment frequently did not normalize their FEV1 or FVC after treatment. One possibility is that mucus plugs are not substantially affected by these treatments and that specific mucoactive drugs will need to be used in these subjects in order to normalize their lung function. The CT mucus score that we describe here will be invaluable in identifying subjects for precision clinical trials of this approach. In this regard, it is important that we discovered that symptoms of $\mathrm{CMH}$ are neither sensitive nor specific for the mucus plug phenotype we uncovered here, perhaps because the mucus plugs occur in subsegmental airways that lack large numbers of cough receptors (19-21). We also show that the clinical and airway inflammation features of the $\mathrm{CMH}$ phenotype are different from the features of the mucus plug phenotype. Thus, not all mucus phenotypes in asthma are the same, and we demonstrate the unique ability of MDCT lung imaging to identify patients with a mucus plug phenotype.

Airway mucus in health is normally a lightly crosslinked gel that does not form plugs $(22,23)$, and it is puzzling why mucus plugs were so common in our subjects. Cellular and molecular analyses of airway biospecimens from subjects with mucus plugs and controls provided valuable clues about the mechanism of mucus plug formation. The main clue came from analysis of sputum leukocytes in which we found that subjects with mucus plugs had marked sputum eosinophilia. They also had marked increases in sputum cell gene expression for type 2 cytokines, such as IL-5 and IL-13, and a relative increase in MUC5AC gene expression over MUC5B. This latter finding is relevant because cysteine domains are more prevalent in MUC5AC than in MUC5B (24), and we have previously shown that neutrophil-driven oxidation in the airways in cystic fibrosis can crosslink cysteine-rich mucin polymers to stiffen the mucus gel (11). In our studies, we found strong evidence that eosinophil-driven oxidation mediates mucus plug formation in asthma. First, we found that EPO levels were high in subjects with mucus plugs and that EPO showed activities in model systems of the airway mucus gel that promoted increased elasticity of the gels. Specifically, we found that EPO catalyzed reactions of $\mathrm{H}_{2} \mathrm{O}_{2}$ with bromide and thiocyanate to generate oxidants (HOBr and HOSCN, respectively) that could convert cysteines to their oxidized disulfide products (cystines). We also found that HOSCN could oxidize a thiolated hydrogel to convert it from a liquid form to a solid form. HOSCN could be expected to form in 
the lumen of asthmatic airways because of the pleotropic effects of type 2 cytokines, which included effects that caused eosinophil accumulation (23), hydrogen peroxide production (25), and pendrin upregulation (26). Pendrin is relevant because it mediates the exchange of thiocyanate across airway epithelial cells (27). Taken together, our data lead us to propose that mucus plugs form in the airways of patients with asthma because of multiple consequences of airway type 2 inflammation that lead to mucin-eosinophil interactions that promote mucus plugs (Figure 7F).

In summary, our data provide strong evidence that airway mucus plugs are common in chronic severe asthma, persist over time, and contribute to mechanisms of chronic airflow obstruction. We also provide data to implicate EPO as a key mediator in generating thiol-specific oxidants that oxidize mucins to cause mucus plugs to form. We propose that the detection of mucus plugs in the lung using MDCT lung scans can serve as a biomarker of disease severity in the airways of patients with asthma and can be indicative of outcome for clinical trials that could determine whether mucolytics or inhibitors of type 2 inflammation can decrease mucus plugs to improve airflow in chronic severe asthma.

\section{Methods}

Subjects. Adult asthma subjects were recruited as part of the SARP 3 cohort. The SARP 3 protocol includes 3 baseline visits in which asthma subjects undergo detailed characterization, including sputum questionnaires, MBRT, a systemic corticosteroid responsiveness test, and an optional MDCT scan of the lungs (Supplemental Figure 6). Data reported here are from subjects that had MDCT scans as part of their baseline characterization. CT was not repeated after steroid injection. Healthy subjects for MDCT scans were recruited at a single center (Washington University), and subjects for sputum cell analyses were recruited from all SARP 3 centers (Supplemental Table 1). Twentyfive asthma subjects who had MDCT scans as part of the SARP 3 protocol also had MDCT scans available from their participation in SARP 1 or SARP 2 protocols. These subjects were enrolled at 3 sites: University of Pittsburgh, University of Wisconsin, and Washington University (Supplemental Table 2). Forty-three asthma subjects who had MDCT scans as part of the SARP 3 protocol also had plethysmography performed. These subjects were enrolled at the University of Wisconsin (Supplemental Table 1).

Sputum and cough questions. Questionnaires were completed by asthma subjects at study entry. CMH was defined using the ATS/ WHO definition of chronic bronchitis, which assesses chronic cough and sputum production in the preceding 2 years (16). The specific question used was the following: Have you had cough and sputum production on most days for at least 3 months a year for at least 2 consecutive years?

$M B R T$. Subjects were asked to hold their bronchodilator medications prior to spirometry testing. Following baseline spirometry, 4 puffs of albuterol $(360 \mathrm{mcg})$ were administered. Spirometry was then repeated 15 minutes later. If the change in FEV1 from the spirometry maneuver performed after 4 puffs was greater than $5 \%$, an additional 2 puffs of albuterol $(180 \mathrm{mcg})$ were then administered and spirometry was repeated again 15 minutes later. If the change in FEV1 after 6 puffs was greater than $5 \%$, an additional 2 puffs of albuterol were administered with repeat spirometry after an additional 15 minutes. If the change was less than $5 \%$ after 4 or 6 puffs of albuterol, the procedure was stopped and the last maneuver was taken to be the highest achievable measure. No more than 8 puffs of albuterol were administered as part of the MBRT procedure. MBRT was measured on baseline visits 2 and 3 (Supplemental Figure 6).

SCRT. Subjects were given an intramuscular injection of triamcinolone acetonide $(40 \mathrm{mg})$ following complete characterization on visit 2. Repeat characterization after steroid injection (excluding MDCT) was carried out on visit 3 (2 to 4 weeks later) (Supplemental Figure 6).

MDCT protocol and analysis. Details about the MDCT parameters are provided in Supplemental Tables 6 and 7. A standard window width of $1200 \mathrm{HU}$ and level of $600 \mathrm{HU}$ were used for visual bronchial wall evaluation (28). Radiologists were blinded to the clinical characteristics of the subjects. Quantitative measures of airway wall thickness and lumen area were generated using Apollo 1.2 (VIDA Diagnostics) (Supplemental Figure 7) and further described in the Supplemental Methods.

Sputum induction, cell counts, and gene expression. Sputum induction was performed on visits 2 and 3 (Supplemental Figure 6). For safety, induced sputum was only collected from subjects whose FEV1 was more than $50 \%$ of predicted volume after albuterol pretreatment (360 ug). Total and differential cell counts and sputum cell gene expression were quantified at a centralized laboratory using methods previously described (29-31). The primers and probes used for measurement of gene expression are provided in Supplemental Table 8.

EPO assay. Human EPO was measured by sandwich ELISA in supernatant collected by sputum induction methods adopted from the NHLBI Asthma Clinical Research Network (32). Samples were diluted 1:50 in assay diluent, per manufacturer-recommended protocols (Diagnostic Development). The detection limit of the assay is less than $0.2 \mathrm{ng} / \mathrm{ml}$.

Cysteine crosslinking assay. To explore cystine formation generated by EPO with $\mathrm{H}_{2} \mathrm{O}_{2}$ in the presence of chloride, bromide, or thiocyanate, BODIPY FL L-cysteine was generated from $800 \mathrm{mM}$ BODIPY FL L-cystine (Thermo Fisher Scientific) in Tyrode's Salts by reduction with one-quarter volume packed TCEP-Gel (Thermo Fisher Scientific) for 1 hour at $25^{\circ} \mathrm{C}$. The reaction yielded an 8- to 10 -fold increase in fluorescence at $490 \mathrm{~nm} / 520 \mathrm{~nm}$ excitation/emission (Ex/Em). This reagent was diluted in $100 \mu \mathrm{l}$ Tyrode's Salts to $4 \mu \mathrm{M}$ with and without PMA (100 ng) in 96-well round-bottom nontreated black polystyrene plates (Corning Costar). The decrease in fluorescence at $490 \mathrm{~nm} / 520$ $\mathrm{nm}$ Ex/Em was monitored over 90 minutes at $37^{\circ} \mathrm{C}$ on a Synergy $\mathrm{H} 1$ plate reader (BioTek Instruments) with the addition of EPO with $\mathrm{H}_{2} \mathrm{O}_{2}$ and a (pseudo)halide. The plates were sealed with optical adhesive film (Applied Biosystems) to prevent evaporation. The quenching of BODIPY fluorescence by eosinophil stimulation was shown to return to baseline values by the addition of DTT to the wells at the end of incubation, indicating that this effect was due to reformation of cystine and not destruction or bleaching of the fluorophore.

Thiolated hydrogel. HOSCN, the product of the EPO-catalyzed reaction of $\mathrm{H}_{2} \mathrm{O}_{2}$ with potassium thiocyanate (KSCN), was examined for its ability to increase the elasticity of thiolated hyaluronic acid. $\mathrm{KSCN}(2 \mathrm{mM})$ and $\mathrm{H}_{2} \mathrm{O}_{2}(2 \mathrm{mM})$ were incubated for 15 minutes at $25^{\circ} \mathrm{C}$ with or without EPO $(6 \mathrm{nM})$ in a $0.5 \%$ solution of thiolated hyaluronic acid/phosphate buffer pH 7.4 (Glycosil, Ascendance Bio). The elastic moduli $\left(G^{\prime}\right)$ for the solutions were then measured on a cone and plate rheometer (TA Instruments) oscillating at $1 \mathrm{~Hz}$ and $5 \%$ strain. The gel solution without reactants was also assessed for background elasticity. 
Statistics. Agreement between raters was estimated using the ICC calculated with a 1-way random effects ANOVA model. Within-rater agreement was calculated using a random subset of 14 scans. Categorical variables are presented as frequencies with percentages and were evaluated using the $\chi^{2}$ test. Continuous variables are presented as mean $\pm 1 \mathrm{SD}$ or medians with quartiles. Box-and-whisker plots were prepared showing the median (marked by a horizontal line), 25\% and $75 \%$ quartiles (box), and $1.5 \times$ interquartile range (IQR) (whiskers). Data points outside 1.5 IQR are plotted as outliers. One-way ANOVA was used for multiple group comparison, followed by Bonferroni's correction. Kruskal-Wallis 1-way ANOVA was used for nonparametric multiple group comparison. Correlation between variables was evaluated using Spearman's correlation. Multivariable analyses were calculated using linear and logistic regression models (with 95\% CIs). Two-sided $P$ values of less than 0.05 were considered statistically significant. Statistical analysis was carried out using Stata 13.1 (StataCorp).

Study approval. Written informed consent approved by each center's institutional review board was received from participants prior to inclusion in the study. Study procedures and sample collection were carried out using standardized protocols approved by each center's institutional review board.

Data availability. Data are available on request from the authors.

\section{Author contributions}

EMD and JVF conceived and designed the study. BME, DSG, SKN, MLS, and JDN contributed significantly to the design of the CT mucus score, validated the score in a subset, and applied the score to the CT scans in the study. SBF played an advisory role in the development of the score, and EAH, MC, SBF, NNJ, EI, BDL, SCE, SEW, DAM, ERB, BRP, DTM, EDG, PGW, and MCP made substantial contributions to the design and analysis of this study. In addition, MCP provided sputum gene-expression analysis.
MELS performed the EPO measurements and made substantial contributions to experimental design. SDM developed the hydrogel model for mucus experiments. WWR designed and carried out the cysteine and hydrogel assays. EMD, JVF, and BRP conducted the data analysis. EMD and JVF prepared the first draft of the manuscript, and all authors revised the draft critically for intellectual content. All authors have given final approval for the manuscript version to be published.

\section{Acknowledgments}

We thank the volunteers who participated in these studies and Patricia Noel and Robert Smith from the Division of Lung Diseases, NHLBI, for their support and leadership of the SARP. We thank the investigators and coordinators in the SARP who contributed to this manuscript by recruiting and characterizing participants, collecting biospecimens, developing systems for lung imaging and analysis, and coordinating data collection and analysis (see Supplemental Acknowledgments). Additional acknowledgments and details of grant funding sources from the National Institutes of Health is also provided in the Supplemental Acknowledgments.

Address correspondence to: John Fahy, Room 1303, Health Sciences East, UCSF, 513 Parnassus Avenue, San Francisco, California 94143, USA. Phone: 415.476.9940; Email: john.fahy@ucsf.edu.

EMD's present address is: School of Medicine, University College Dublin, Dublin, Ireland.

DAM and ERB's present address is: Division of Genetics, Genomics and Precision Medicine, Department of Medicine, University of Arizona, Tucson, Arizona, USA.
1. Huber HC, Koessler KK. The pathology of bronchial asthma. Arch Intern Med.1922;30(6):689-760.

2. Dunnill MS. The pathology of asthma, with special reference to changes in the bronchial mucosa. JClin Pathol. 1960;13:27-33.

3. Vestbo J, Prescott E, Lange P. Association of chronic mucus hypersecretion with FEV1 decline and chronic obstructive pulmonary disease morbidity. Copenhagen City Heart Study Group. Am J Respir Crit Care Med. 1996;153(5):1530-1535.

4. Ulrik CS, von Linstow ML, Nepper-Christensen S, Porsbjerg C, Backer V. Chronic mucus hypersecretion: a marker of asthma in young adults? Respir Med. 2005;99(12):1576-1582.

5. Burgel PR, Martin C. Mucus hypersecretion in COPD: should we only rely on symptoms? Eur Respir Rev. 2010;19(116):94-96.

6. Boser SR, Park H, Perry SF, Ménache MG, Green FH. Fractal geometry of airway remodeling in human asthma. Am J Respir Crit Care Med. 2005;172(7):817-823.

7. Fabbri LM, et al. Differences in airway inflammation in patients with fixed airflow obstruction due to asthma or chronic obstructive pulmonary disease. Am J Respir Crit Care Med. 2003;167(3):418-424.

8. Bumbacea D, et al. Parameters associated with persistent airflow obstruction in chronic severe asthma. Eur Respir J. 2004;24(1):122-128.

9. Henderson WR, Chi EY, Klebanoff SJ. Eosinophil peroxidase-induced mast cell secretion. JExp Med. 1980;152(2):265-279.

10. Arlandson M, et al. Eosinophil peroxidase oxidation of thiocyanate. Characterization of major reaction products and a potential sulfhydryltargeted cytotoxicity system. J Biol Chem. 2001;276(1):215-224.

11. Yuan $S$, et al. Oxidation increases mucin polymer cross-links to stiffen airway mucus gels. Sci Transl Med. 2015;7(276):276ra27.

12. Carr TF, Berdnikovs S, Simon HU, Bochner BS, Rosenwasser LJ. Eosinophilic bioactivities in severe asthma. World Allergy Organ J. 2016;9:21.

13. Chung KF, et al. International ERS/ATS guidelines on definition, evaluation and treatment of severe asthma. Eur Respir J. 2014;43(2):343-373.

14. Sorkness RL, et al. Lung function in adults with stable but severe asthma: air trapping and incomplete reversal of obstruction with bronchodilation. J Appl Physiol. 2008;104(2):394-403.

15. Agarwal R, et al. Allergic bronchopulmonary aspergillosis: review of literature and proposal of new diagnostic and classification criteria. Clin Exp Allergy. 2013;43(8):850-873.

16. American Thoracic Society. Definitions and classification of chronic bronchitis, asthma and pulmonary emphysema. Am Rev Respir Dis. 1962;85:762-768.

17. Bonser LR, Zlock L, Finkbeiner W, Erle DJ. Epithelial tethering of MUC5AC-rich mucus impairs mucociliary transport in asthma. J Clin Invest. 2016;126(6):2367-2371.

18. Shu XZ, Liu Y, Luo Y, Roberts MC, Prestwich GD. Disulfide cross-linked hyaluronan hydrogels. Biomacromolecules. 2002;3(6):1304-1311.

19. [No authors listed]. Cough and expectoration. Paris, October 3-4, 1979. Eur JRespir Dis Suppl. 1980;110:1-262.

20. Jackson C. Cough: Bronchoscopic observations on the cough reflex. JAMA. 1922;79(17):1399-1403.

21. Widdicombe JG. Neurophysiology of the cough reflex. Eur Respir J. 1995;8(7):1193-1202.

22. Innes AL, et al. Ex vivo sputum analysis reveals impairment of protease-dependent mucus degradation by plasma proteins in acute asthma. Am J Respir Crit Care Med. 2009;180(3):203-210.

23. Fahy JV, Dickey BF. Airway mucus function and dysfunction. NEnglJMed.2010;363(23):2233-2247.

24. Thornton DJ, Rousseau K, McGuckin MA. Structure and function of the polymeric mucins in airways mucus. Annu Rev Physiol. 2008;70:459-486.

25. Rada B, Leto TL. Characterization of hydrogen peroxide production by Duox in bronchial epithelial cells exposed to Pseudomonas aeruginosa. 
FEBS Lett. 2010;584(5):917-922.

26. Zhen G, et al. IL-13 and epidermal growth factor receptor have critical but distinct roles in epithelial cell mucin production. Am J Respir Cell Mol Biol. 2007;36(2):244-253.

27. Pedemonte N, et al. Thiocyanate transport in resting and IL-4-stimulated human bronchial epithelial cells: role of pendrin and anion channels. J Immunol. 2007;178(8):5144-5153.

28. Bankier AA, et al. Bronchial wall thickness: appropriate window settings for thin-section CT and radiologic-anatomic correlation. Radiology. 1996;199(3):831-836.

29. Gershman NH, Wong HH, Liu JT, Mahlmeister MJ, Fahy JV. Comparison of two methods of collecting induced sputum in asthmatic subjects. Eur Respir J. 1996;9(12):2448-2453.

30. Hastie AT, et al. Biomarker surrogates do not accurately predict sputum eosinophil and neutrophil percentages in asthmatic subjects. JAllergy
Clin Immunol. 2013;132(1):72-80.

31. Peters MC, Mekonnen ZK, Yuan S, Bhakta NR, Woodruff PG, Fahy JV. Measures of gene expression in sputum cells can identify TH2-high and TH2-low subtypes of asthma. J Allergy Clin Immunol. 2014;133(2):388-394.

32. Fahy JV, et al. Safety and reproducibility of sputum induction in asthmatic subjects in a multicenter study. Am J Respir Crit Care Med. 2001;163(6):1470-1475. 